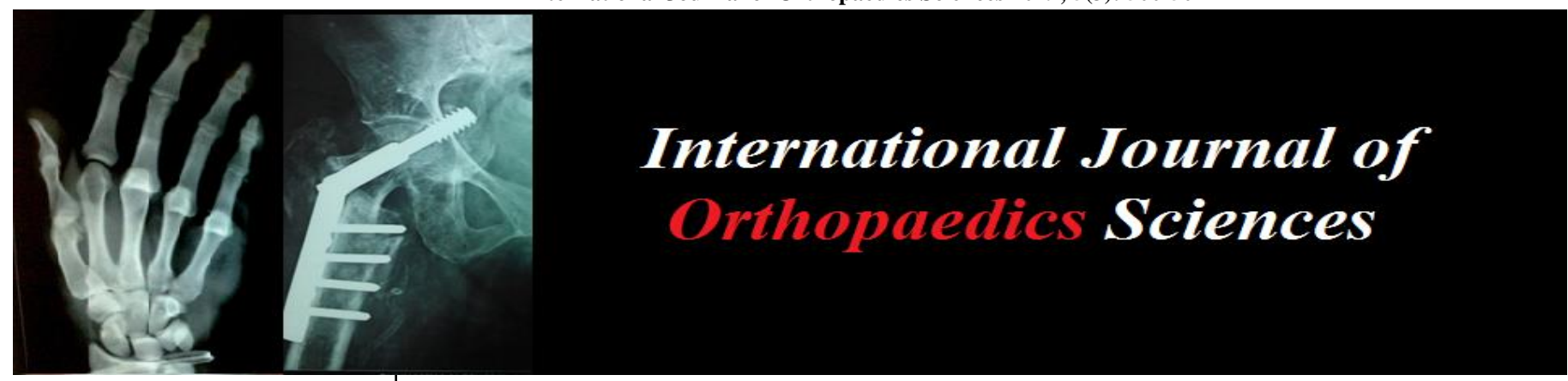

ISSN: $2395-1958$

IJOS 2019; 5(3): 500-502

(C) 2019 IJOS

www.orthopaper.com

Received: 01-05-2019

Accepted: 03-06-2019

Dr. Ravikiran Lagali

Pandit Dindayal Upadhyay

Medical College, Sadar Civil

Hospital Campus, Gujarat, India

\section{Results of distal femur fracture treated by distal femur locking plates: A study of 30 cases}

\section{Dr. Ravikiran Lagali}

DOI: https://doi.org/10.22271/ortho.2019.v5.i3i.1576

\begin{abstract}
Supracondylar and intercondylar fractures of the distal femur fracture historically have been difficult to treat. These fracture often unstable and comminuted and tend to occur in elderly or those with multiple injuries. Treatment options available today are many with varied results. The final outcome would depend on the type of fracture, stabilization and fixation and perhaps condition of skin and soft tissue. Since the introduction of the locking compression plates which has been available for the clinical use since 2001, it has revolutionized internal phase so for this system combines two different principle of fixation. This study deals with clinical and radiological outcomes of distal femur fractures treated with locking compression plate and compare the results achieved with treatment results available in the literature.
\end{abstract}

Keywords: Distal femur fractures, locking compression plate, distal femur locking plate

\section{Introduction}

Fracture of distal femur accounts for 7 percent of all femoral fractures and 30 percent of fractures of femur excluding hip fractures. Distal femoral fractures mainly arise from two different injury mechanisms ${ }^{[1]}$. High energy trauma mainly sustained in road traffic accidents in younger patient ${ }^{[2]}$. Low energy trauma, in elderly patients with severe osteoporosis. Since last two decades incidence of this type of fractures has increased a lot due to a rise in road traffic accidents. Distal femur fractures present considerable challenge in management and outcome. Before 1970 Studies advised conservative treatment for distal femur fractures. Later studies advised operative treatment and angular blade plate had significantly higher torsional stiffness than other constructs. Locking compression Plate evolved from conventional plates and is widely used nowadays because of biomechanical advantage. 1,2 The principle of the Locking compression plate is to have rigid fixation close to the bone and under the soft-tissue envelope and can be applied without stripping periosteum which is very much essential for fracture healing. Distal femur locking plates can be applied in three different ways: as a conventional dynamic compression plate providing absolute stability.

In combined fashion where both techniques are employed using conventional lag screw as well as locked screws. The implant offers multiple points of fixed-angle contact between the plate and screws in the distal part of femur, theoretically reducing the tendency for Varus collapse that is seen with traditional lateral plates. The purpose of this study is to evaluate the outcome, effectiveness and complications of distal femur fractures, treated by open reduction and internal fixation using distal femoral locking compression Plate.

\section{Objectives}

To study the functional outcome for internal fixation of the distal femur fracture by distal femur locking plate To evaluate the effectiveness of distal femur fractures treated with locking compression plate.

\section{Methodology}

The study was conducted on patients of distal femur fracture treated by locking compression plate in Department of Orthopedics, Pandit Deen Dayal Upadhyay Medical College, during the study period which extended from December 2014 to June 2016. 
All the patients during the study period were included in the study which included 30 patients after considering inclusion and exclusion criteria. The patients were followed up and data were collected by clinical examination, Neers' scoring6 and X rays taken immediately after operation, at 6 weeks, 12 weeks and 24 weeks after surgery.

\section{Inclusion criteria consisted of}

- All patients with distal femur fractures treated with DFLP

- All skeletal mature patients (>18years).

- Patients willing to give consent

- Closed fracture with normal dnv

\section{Exclusion Criteria}

- Patients of age less than 18 years

- Open fractures type III B and C

- Pathological Fractures

- Associated tibial plateau fractures

- Compound and extensive soft tissue injuries

- Compromised distal neuro-vascularity

- Ipsilateral fracture neck femur

This study was approved by ethical committee and also the consent was obtained from the patients before collection of data. Statistical tests were applied wherever necessary. Microsoft excel was used for descriptive statistics.

\section{Results}

The study included 30 patients who majorly 15 (50.0\%) fall in the age group of 20-45 years. The mean age group of the patients was found to be 42 years with a standard deviation of 12 years. Table no 1 shows in detail the age distribution of patients. There were majority males $22(73.3 \%)$ among the study. The male: female ratio was found to be $2.7: 1$.

Table 1: Age-wise distribution of Patients

\begin{tabular}{|c|c|c|}
\hline Age group & Number (N) & Percentage (\%) \\
\hline$<20$ & 2 & 6.6 \\
\hline $20-45$ & 15 & 50 \\
\hline $45-60$ & 12 & 40 \\
\hline$>60$ & 1 & 3.4 \\
\hline
\end{tabular}

Majority $57 \%$ of the patients had injury duty to road traffic accident. (Figure 1). Majority of the fractures were extraarticular $24(80 \%)$ followed by par articular $4(13.3 \%)$, then intraarticular $2(6.6 \%)$.

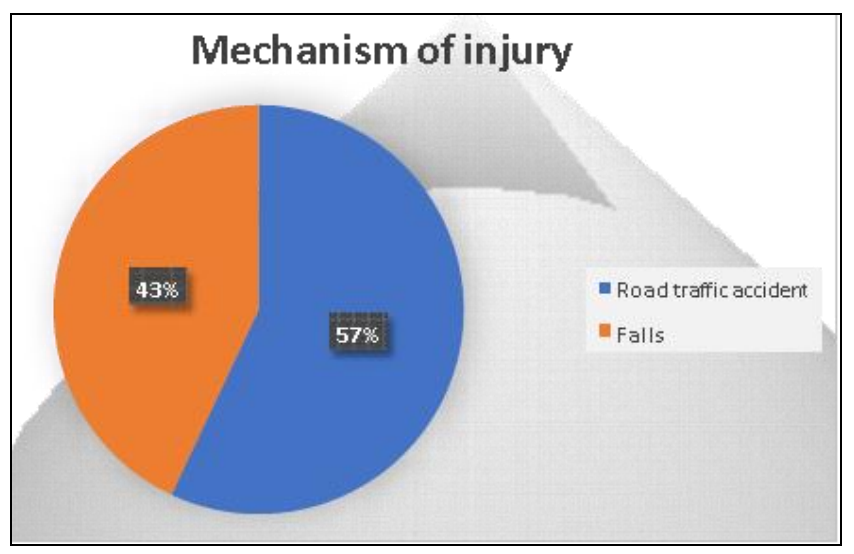

Fig 1: Mechanism of injury of patients.

While seeing the outcome we accessed the radiological union which is accessed by presence of bridging callus across three cortices. The average time for union was found to be 16 weeks with a standard deviation of 1.5 weeks. For effectiveness we studied knee flexion. The current study showed average knee flexion of 110 degrees, more than 110 degrees was present in only 1 patient. The Neer's Score in the study group showed $10 \%$ excellent results followed by $60 \%$ had good results and there were $30 \%$ who had failure. The study also saw for complications there were only $3(10 \%)$ complications.

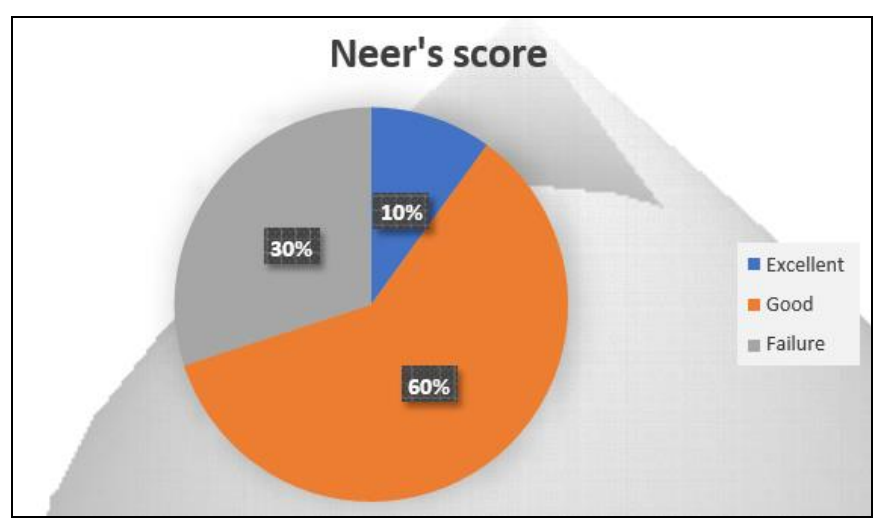

Fig 2: Distribution of patients according to Neer's 3 score

\section{Discussion}

Distal femoral fractures are challenging injuries despite improvements of fixation techniques and plate designs have demonstrated the ability of locked plates to absorb more energy before failure compared with angled blade plates or retrograde intramedullary nails, thereby having a lower incidence of loss of fixation. Although no agreement exists on management of complex distal femoral fractures, the results reported by several suggest modern locking plates represent an advance for fixing different fracture patterns in this region. These include either high-energy fractures with severe bone comminution that may be further complicated through open injury, fractures in older people with poor bone quality and peri prosthetic fractures.

The disadvantages of first-generation locked plates include the uniaxial screw trajectories. These screws trajectories cannot account for differences in femoral anatomy, fracture patterns or variations in plate positioning. Treatment of comminuted supracondylar fractures with the loss of medial buttress (AO type $33 \mathrm{~A} 3$ ) present a challenging problem for orthopedic surgeons and are one of the most debated topics recently. Numerous implants have been designed for distal femur fracture fixation such as ABP, CBP, DCS, cancellous screws, LCP, retrograde interlocking nail and ante grade interlocking nail IMN. For last 30 years, DCS and ABP has been most favored implants, but at present locking plates (DFLP) and less invasive stabilization system (LISS) are being used more commonly.

This study comprised of 30 cases with distal femur fractures treated with distal femur locking plate. In this study 22 were male and 8 were females. The median age group was 40 ranging from 18 to 65 years. In $57 \%$ of the study group mechanism of injury was road traffic accident while in $43 \%$ it was domestic fall. Road traffic accident are more common in younger males and domestic fall more common in elderly females.

Comparing our results with standard studies, in a study by Schutz m, Muller et al ${ }^{[4,5]}$ a series of 62 patients studied and did follow up, mean duration surgery is $85 \mathrm{~min}$ with average time of admission operation interval is 5 days, mean age is 52 
years and non union $5.5 \%$ of cases with malalignment 10 20degree in 2 cases. In present study 30 cases with average time interval of operation is 3 days with mean age 40years, non union $3.3 \%$ of cases with 5 cases $(16 \%)$ required bone grafting.

Markmiller ${ }^{[6]}$ et al prospectively compared outcome of LISS and intramedullary nailing. At 12 months no statically significant differences were noted for nonunion fixation failure, infection, and secondary surgical procedures. However this was relatively small series and no power analysis is reported.

Dr. D.S. Ramnath ${ }^{[7]}$ et al. All patients with supracondylar femoral fracture treated with locking plate and age older than 18 years were included in this study. Patients with, metastatic bone disease, nerve injury were excluded.30 fractures were surgically treated for distal femur fractures during the study period. Follow-up was 24 months. Patients were followed at intervals of 2 weeks, 6 weeks, 12 weeks, 6 months, 1 year, and 2 years. Complaints of pain were assessed with a visual analog scale (VAS). Complications were recorded - screw loosening, implant failure, and Revision surgery. Infection was defined as either deep or superficial.

In this study 16 cases were of left side while 14 cases were of right in 30 patients with a mean age of 55 years (range 18-85 years). There were 16 males and 14 females. Surgical complications were found in $20 \%$ of patients. $20 \%$ of the patients developed a non-union. 10\% developed implant failure. $2 \%$ cases developed infection. Neer scoring $20 \%$ excellent, $65 \%$ good and $15 \%$ shows good results. In current study 30 cases male were 22 and 8 were female with mean age of 40 years, 20 cases right limb and 10 cases have left limb. Average range of motion is 101.3 degree. Neer score showing $10 \%$ excellent, $60 \%$ good, $30 \%$ fair results.

\section{Conclusion}

1. The introduction of locking plates with option of locked screws has provided the means to increase the rigidity of fixation.

2. Operative time duration can be significantly with locking compression plate as it requires less surgical dissection. Surgical time much reduced with mippo technique.

3. The device produce good angular stability by its triangular reconstruction principle. Also helps in early mobilisation.

4. By mippo technique soft tissue disturbance is very less with lesser complications.

5. Potential reasons for the implant failure include technical errors in plate placement and early weight bearing im the presence of delayed fracture union

\section{References}

1. Gross AE. Periprosthetic fractures of the knee: puzzle pieces. J Arthroplasty 2004; 4(1):47-50.

2. Kim KI, Egol KA, Hozack WJ, Parvizi J. Periprosthetic fractures after Total knee arthroplasties. Clin Orthop Relat Res 2006; 446:167-75.

3. Neer CS, Grantham SA, Shelton ML. Supracondylar fracture of the adult femur. A study of one hundred and ten cases. J Bone Joint Surg Am. 1967; 49(4):591-613.

4. Kaab MJ, Stockle U, Schutz M, Stefansky J, Perka C, Haas NP. LISSS tabilization of periprosthetic fractures with angular stable internal fixation: a report of 13 cases. Arch Orthop Trauma Surg. 2006; 126-2:105-10.

5. Muller ME, Allgower M, Schneider R, Willenegger $\mathrm{H}$. Manual of Internalfixation, 1991.
6. Markmiller M, Konrad G, Sudkamp N. Femur-LISS and distal femoral nail for fixation of distal femoral fractures: are there Differences in outcome and complications. Clin Orthop Relat Res. View Article Pub Med Google Scholar. 2004; 426:252-257. 